\title{
Comparison between morphological and staining characteristics of live and dead eggs of Schistosoma mansoni
}

\author{
AK Sarvel, JR Kusel*, N Araújo, PMZ Coelho/**, N Katz**/+ \\ Laboratório de Esquistossomose, Centro de Pesquisas René-Rachou-Fiocruz, Av. Augusto de Lima 1715, 31190-002 \\ Belo Horizonte, MG, Brasil *University of Glasgow, Scotland, UK **Santa Casa de Misericórdia de Belo Horizonte, Belo \\ Horizonte, MG, Brasil
}

Schistosoma mansoni eggs are classified, according to morphological characteristics, as follows: viable mature and immature eggs; dead mature and immature eggs, shells and granulomas. The scope of this study was to compare the staining characteristics of different morphological types of eggs in the presence of fluorescent labels and vital dyes, aiming at differentiating live and dead eggs. The eggs were obtained from the intestines of infected mice, and put into saline $0.85 \%$. The fluorescent labels were Hoechst 33258 and Acridine Orange + Ethidium Bromide and vital dyes (Trypan Blue 0.4\% and Neutral Red 1\%). When labelled with the probe Hoechst 33258, some immature eggs, morphologically considered viable, presented fluorescence (a staining characteristic detected only in dead eggs); mature eggs did not present fluorescence, and the other types of dead eggs, morphologically defined, showed fluorescence. As far as Acridine Orange + Ethidium Bromide are concerned, either the eggs considered to be live, or the dead ones, presented staining with green color, and only the hatched and motionless miracidium was stained with an orange color. Trypan Blue was not able to stain the eggs, considered to be dead but only dead miracidia which had emerged out of the shell. Neutral Red stained both live and dead eggs. Only the fluorescent Hoechst 33258 can be considered a useful tool for differentiation between dead and live eggs.

Key words: Schistosoma mansoni eggs - fluorescent labels - vital dyes

The pathology of the disease is mainly related to the presence of Schistosoma mansoni eggs and their excretion products in the host's tissues. The liver is the organ that shows the most important changes caused by the disease. It is possible to obtain a precise diagnosis of the disease through identification of the eggs. Females lay about 300 eggs/day, as it has been observed in infected rodents. The eggs laid are immature at the first stage, and become mature at the second, third and fourth stages, after approximately six days. When they are not discharged in the feces, they die 12 days after maturation (Prata 1957).

The oogram is generally used for obtaining microscopic counts of $S$. mansoni eggs, including the different evolutive stages. This method is utilized for selecting new drugs, since their activity can be determined few days after the end of treatment (Pellegrino et al. 1962).

While it is easy to distinguish live mature eggs from dead mature ones, either by the movement of the miracidium or by its flame cell, the characterization of the viability of immature eggs is very difficult.

The scope of this study was to compare the staining behavior of different stages of $S$. mansoni.

Financial support: Centro de Pesquisas René-Rachou-Fiocruz, $\mathrm{CNPq}$

${ }^{+}$Corresponding author: nkatz@cpqrr.fiocruz.br

Received 25 May 2006

Accepted 26 June 2006

\section{MATERIALS AND METHODS}

Preparation of eggs - The eggs were obtained from the intestines of Swiss mice (maintained at the animal house of the Research Center René-Rachou-Fiocruz), after 48-56 days of $S$. mansoni infection. Mice were sacrificed by cervical fracture, and the intestines were removed, washed in saline solution $0.85 \%$ and homogeneized. After homogenization of the intestines, the obtained suspension was put into a sedimentation glass, in the dark, for $1 \mathrm{~h}$. The liquid portion was discarded, the sediment washed $1 \times$ in physiological solution, centrifuged for $30 \mathrm{~s}$, and the supernatant was also discarded. Afterwards, the sediment containing eggs was placed into tubes with $1 \mathrm{ml}$ saline $0.85 \%$ (Pellegrino \& Katz 1968).

Criteria for classification of eggs - The eggs were classified as follows: (1) viable immature eggs (1st to 4th stages) and mature ones; (2) dead immature eggs (semitransparent, darkened, granular, and eggs with retracted embryo) and dead mature ones (calcified, eggs with disintegrating miracidium, eggs with retracted miracidium, and roughly granulated eggs); (3) shells; and (4) granulomas (Pellegrino et al. 1962).

Controls - Positive and negative controls for the experiment were performed, as well as suspension that was used with the labels and dyes. The positive control consisted of mature eggs, which were observed under optical microscopy, in order to verify if there was any movement of the miracidium, the flame cells, as well as the hatching of eggs.

The negative control were eggs after by 1-day-freezing at $-20^{\circ} \mathrm{C}$. After that, the suspension with eggs was thawed and the dye was added. 
Fluorescent labels - The following labels were utilized: Hoechst 33258 (bisbenzamide) (2.4 hydroxyphenil 5.4 methyl, 1 piperazine 2.5 bi H-benzymidazol), which is a hydrophilic and fluorescent probe only when bounded the DNA of cells. However, since it is hydrophilic, it can diffuse in the inner of the cells only in the presence of lesions, acting as an indicator of the membrane integrity. This probe was diluted into saline $0.85 \%$, and $1 \mathrm{mg} / \mathrm{ml}$ of stock solution was obtained. For each $1 \mathrm{ml}$ of egg suspension already tested, $10 \mu \mathrm{l}$ probe were added. The prepared slides were kept at room temperature, for $20 \mathrm{~min}$, and observed under fluorescence microscopy with reading filter $460 \mathrm{~nm}$ (Oliveira et al. 2006).

Acridine Orange + Ethidium Bromide are suitable labels for evaluation of cellular viability. The living cells give a green fluorescence with Acridine Orange and the dead cells give an orange fluorescence with Ethidium Bromide. A stock solution $100 \times$ was prepared by dissolving $50 \mathrm{mg}$ Ethidium Bromide and $15 \mathrm{mg}$ of Acridine Orange into $1 \mathrm{ml}$ ethanol $95 \%$ and $49 \mathrm{ml}$ distilled water. This solution was distributed into Eppendorf tubes, with $1 \mathrm{ml}$ each aliquot, and then were frozen. An aliquot was diluted in PBS $(1 \mathrm{x})$ and stocked into an amber glass at $4^{\circ} \mathrm{C}$. For each $500 \mu \mathrm{l}$ egg suspension tested, $25 \mu \mathrm{l}$ of the label were added. The slides prepared were observed under a fluorescence microscope with reading filter $515 \mathrm{~nm}$ (Parks et al. 1979).

Vital dyes - Trypan Blue 0.4\% and Neutral Red 1\% were used separately. Trypan Blue is able to stain only those cells considered dead. For each $500 \mu \mathrm{l}$ of egg suspension, $10 \mu 1$ was added. Neutral Red was chosen, since it was able to stain living cells, and $10 \mu 1$ was also added for each $500 \mu \mathrm{l}$ of egg suspension. The prepared slides were put aside for $5 \mathrm{~min}$ for penetration of dyes, and observed under an optical microscope with 100 and $400 \times$ magnification.

\section{RESULTS}

When labelled with the fluorescent probe Hoechst 33258 , a few eggs morphologically considered as viable immature (Fig. 1A, B) showed fluorescence, but others did not show fluorescence (Fig. 1C, D). The dead eggs (Fig. 1E, F) as well as miracidium outside the shells, without movement (Fig. 1G, H)) showed fluorescence. The live mature eggs, did not show fluorescence (Fig. 1I, J). In relation to Acridine Orange + Ethidium Bromide solution (Fig. 2), only the hatched and motionless miracidium (Fig. 2A, B) was stained with an orange color. Either the eggs considered to be dead (Fig. 2C, D, E, F) or the eggs considered to be alive presented staining with a green color. The live miracidium (Fig. 2G) was stained with a green color. It is worthwhile to note that eggs that were seen stained with a green color under the microscope, lost their initial color and could be seen stained with orange color, when photographed. Trypan Blue (Fig. 3) did not stain the eggs considered to be dead (Fig. 3B, C), but stained motionless miracidia out of the shell (Fig. 3A). Conversely, Neutral Red (Fig. 4) penetrated either the eggs considered to be dead (Fig. 4A) or those alive (Fig. 4C), as well as miracidia out of the shell (Fig. 4B) which were in motion or motionless (Fig. 4D).
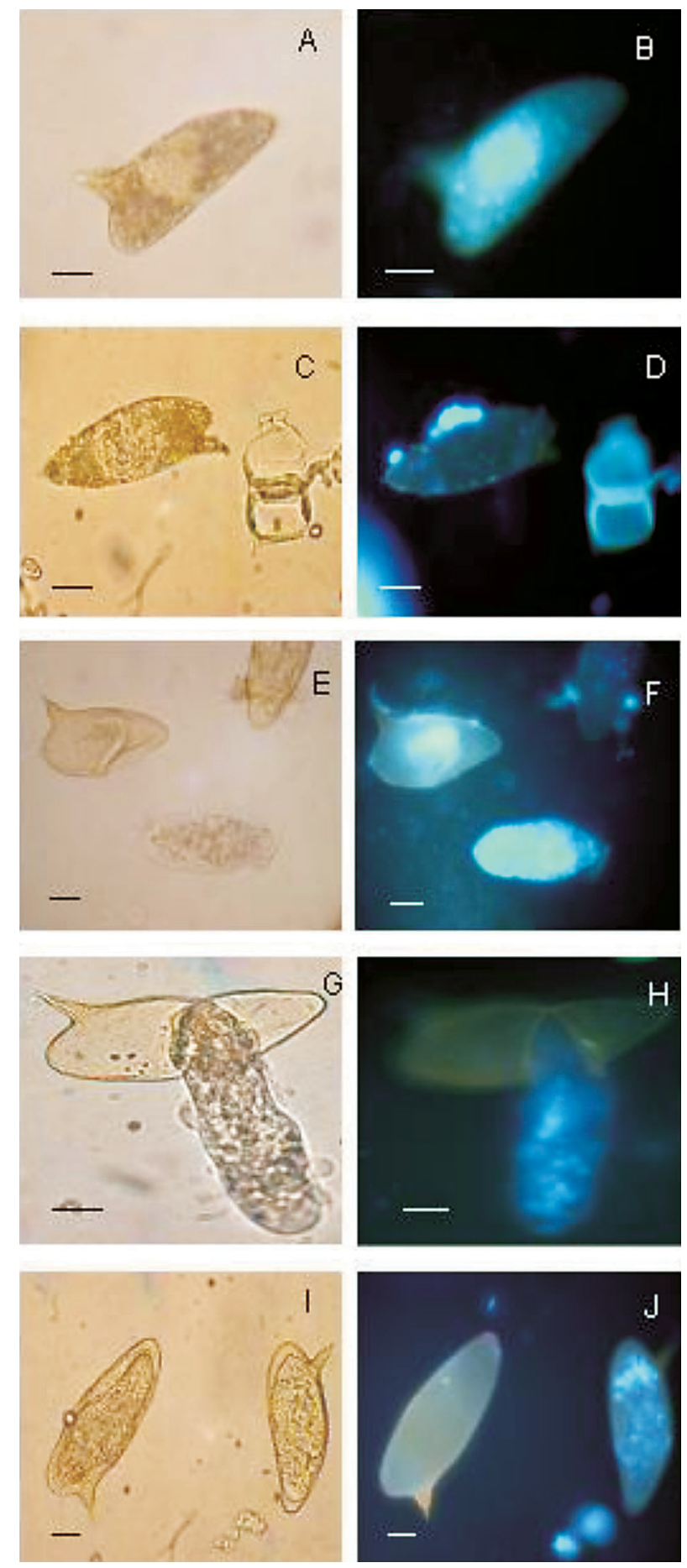

Fig. 1: eggs labeled with Hoechst 33258. A: second stage immature egg, considered viable morphologically by optical microscopy; B: the same egg showing fluorescence; $\mathrm{C}$ : third stage immature egg, considered viable morphologically by optical microscopy; D: the same egg without fluorescence; E: left side empty egg with vitteliginic cells; center dead miracidium; right side, live mature egg, at optical microscopy; F: the same eggs, after fluorescent staining, note that only the live mature egg did not show fluorescence; G: dead miracidium (motionless), by optical microscopy; H: same dead miracidium showing blue fluorescence; I: left side a live mature egg and right side dead mature egg; J: sam eggs at left side without fluorescence and at right with fluorescence. Bars $=30 \mu \mathrm{m}$. 

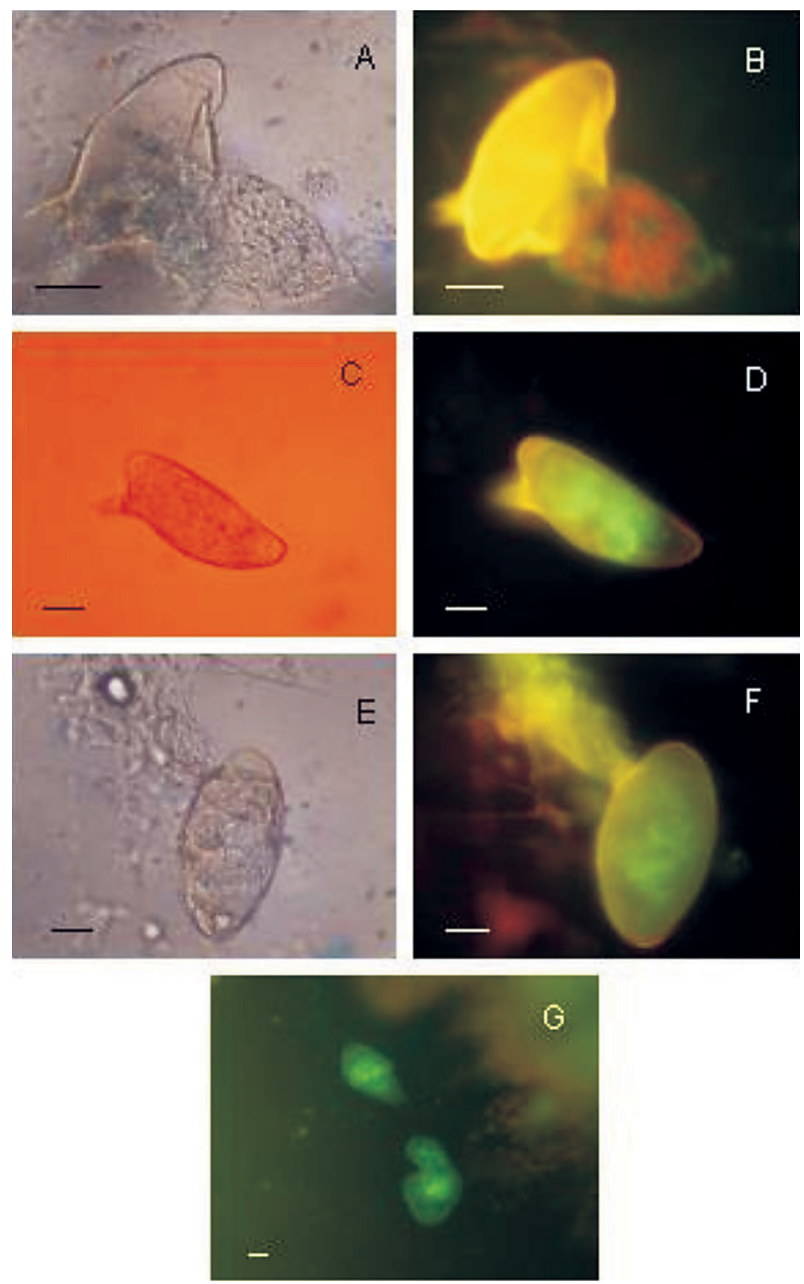

Fig. 2: eggs labeled with Acridine Orange + Ethidium Bromide. A: dead miracidium after hatching by optical microscopy; B: the same miracidium stained with orange colour; $\mathrm{C}$ and $\mathrm{E}$ : eggs with dead embryos by optical microscopy; D and F: same eggs giving green fluorescence; G; two live miracidia giving green fluorescence. Bars $=30 \mu \mathrm{m}$.
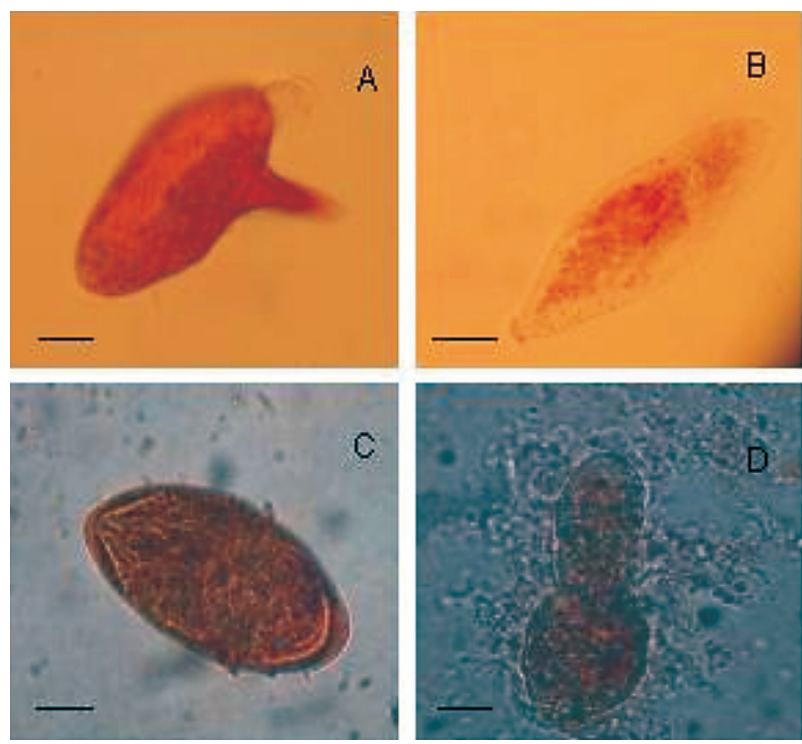

Fig. 4: eggs stained by Neutral Red. A: semitransparent considered to be morphologically dead egg stained by Neutral Red; B: dead miracidium stained by Neutral Red; C: live mature egg stained by Neutral Red; D: live miracidium after hatching, stained by Neutral Red. Bars $=30 \mu \mathrm{m}$

\section{DISCUSSION}

Some eggs considered morphologically viable immature showed fluorescence, when labelled with the probe Hoechst 33258. This could be a result of the presence of lesions morphologically imperceptible under optical microscopy. The eggs morphologically classified as live mature did not show fluorescence, in contrast to the dead mature ones. Taking into account this result, the probe Hoechst 33258 may be considered a good label for membrane integrity. This kind of observation was possible only by means of fluorescence microscopy, since the conventional optical microscopy does not enable to distin-
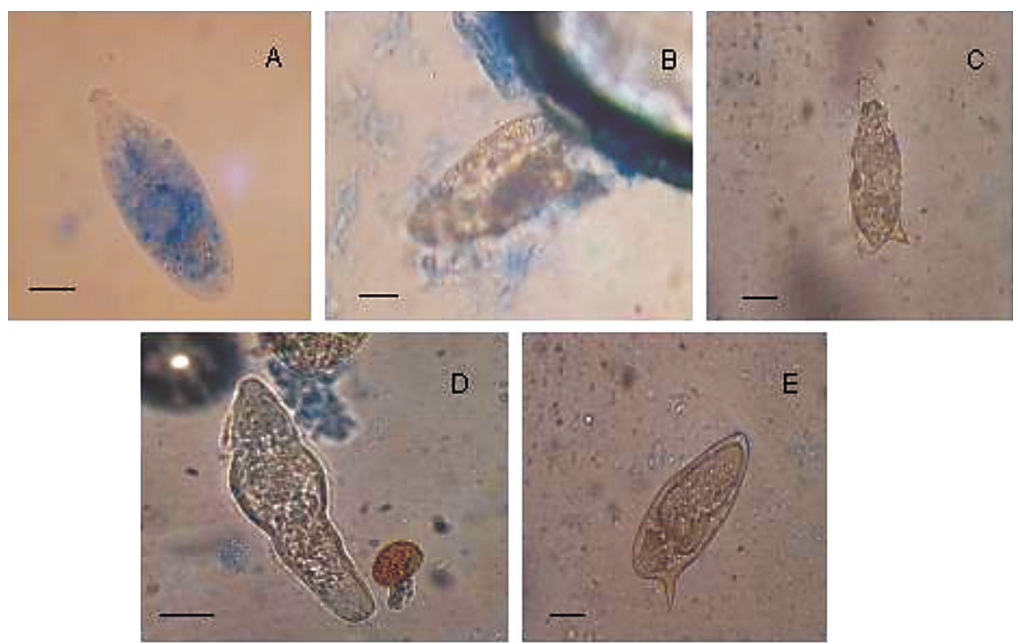

Fig. 3: eggs stained by Trypan Blue. A: blue stained dead miracidium after hatching; B: semitransparent egg not stained by Trypan Blue; C: miracidium retained in egg morphologically dead egg stained by Trypan Blue; D; live miracidium after hatching, not stained by Trypan Blue; E: live mature egg not stained by Trypan Blue. Bars $=30 \mu \mathrm{m}$. 
guish live immature eggs from dead immature ones (Fig. $1 \mathrm{~A}, \mathrm{~B}, \mathrm{C}, \mathrm{D})$.

Acridine Orange + Ethidium Bromide were not able to distinguish between live and dead eggs, since either the live eggs or the dead ones that were expected to be stained with green and orange colors, respectively, could be seen stained with green color and only the miracidium which was dead and out of the shell presented orange staining. This was inconsistent with other evidence of viability. It is also interesting to note that the egg could be seen stained with a green color under the microscope, but when photographed it showed an orange color. Considering these results, it can be concluded that those dyes were not good labels to differentiate cellular viability in eggs.

The vital dyes used were not good tools to differentiate live and dead eggs, since Trypan Blue did not stained the eggs considered to be dead morphologically. This dye is largely used in experiments that require confirmation of viability of mammalian cells. On the other hand, it was possible to observe staining of miracidium out of the shell. This could be perhaps explained considering the larger size of the dye molecule in relation to the pore of the egg, thus impairing Trypan Blue penetration. Neutral Red stained either live and dead eggs, as well as live and dead miracidia out of the shell, and this was not expected. With the results obtained with the vital dyes used in this experiment, it can be concluded that both were not effi- cient in differentiating between live and dead eggs. Only, the fluorescence Hoechst 33258 can be considered a good tool for differentiation between dead and alive S. mansoni eggs.

\section{ACKNOWLEDGEMENTS}

To Prof. Symone Fulgêncio Lima, PUC/MG, and to the technicians of the Laboratory of Schistosomiasis, Aureo Almeida and Daniela Nabak, for assistance with the experiments.

\section{REFERENCES}

Oliveira FA, Kusel JR, Ribeiro F, Coelho PMZ 2006. Responses of the surface membrane and excretory system of Schistosoma mansoni to damage to treatment with praziquantel and other biomelecules. Parasitology 132: 321330.

Parks DR, Bryan VM, OI VM, OI VT, Herzenberg LA 1979. Antigen specific identification and cloning of hybridomas with a fluorescence activated cell sorter (FACS). PNAS 76: 1962.

Prata A 1957. Biópsia Retal na Esquistossomose Mansoni. Bases e Aplicações no Diagnóstico e Tratamento, Thesis, Serviço Nacional de Educação Sanitária, Rio de Janeiro, 197 pp.

Pellegrino J, Katz N 1968. Experimental chemotherapy of Schistosoma mansoni. Adv Parasitol 6: 233-291.

Pellegrino J, Oliveira C A, Faria J, Cunha A S 1962. New approach to the screening of drugs in experimental schistosomiasis mansoni in mice. Amer J Trop Med Hyg 11: 201215 . 